\title{
Transcriptomic and anatomic profiling reveal the germination process of different wheat varieties in response to waterlogging stress
}

Changwei Shen ${ }^{1}$, Jingping Yuan², Hong Qiao ${ }^{3}$, Zijuan Wang ${ }^{3}$, Yuanhai Liư ${ }^{3}$, Xiujuan Ren ${ }^{1}$, Fei Wang ${ }^{1}$, Xing Liu', Ying Zhang ${ }^{1}$, Xiling Chen ${ }^{1}$ and Xingqi Ou ${ }^{4^{*}}$ (D)

\begin{abstract}
Background: Waterlogging is one of the most serious abiotic stresses affecting wheat-growing regions in China. Considerable differences in waterlogging tolerance have been found among different wheat varieties, and the mechanisms governing the waterlogging tolerance of wheat seeds during germination have not been elucidated.

Results: The results showed no significant difference between the germination rate of 'Bainong 207' (BN207) (after $72 \mathrm{~h}$ of waterlogging treatment) and that of the control seeds. However, the degree of emulsification and the degradation rate of endosperm cells under waterlogging stress were higher than those obtained with the control treatment, and the number of amyloplasts in the endosperm was significantly reduced by waterlogging.

Transcriptomic data were obtained from seed samples (a total of 18 samples) of three wheat varieties, 'Zhoumai 22' (ZM22), BN207 and 'Bainong 607' (BN607), subjected to the waterlogging and control treatments. A comprehensive analysis identified a total of 2775 differentially expressed genes (DEGs). In addition, an analysis of the correlations among the expression difference levels of DEGs and the seed germination rates of the three wheat varieties under waterlogging stress revealed that the relative expression levels of 563 and 398 genes were positively and negatively correlated with the germination rate of the wheat seeds, respectively. Gene Ontology (GO) and Kyoto Encyclopedia of Genes and Genomes (KEGG) analyses showed that the difference in the waterlogging tolerance among the three wheat varieties was related to the abundance of key genes involved in the glycolysis pathway, the starch and sucrose metabolism pathway, and the lactose metabolism pathway. The alcohol dehydrogenase (ADH) gene in the endosperm of BN607 was induced immediately after short-term waterlogging, and the energy provided by the glycolysis pathway enabled the BN607 seeds to germinate as early as possible; in addition, the expression of the AP2/ERF transcription factor was upregulated to further enhance the waterlogging tolerance of this cultivar.
\end{abstract}

Conclusions: Taken together, the results of this study help elucidate the mechanisms through which different wheat varieties respond to waterlogging stress during germination.

Keywords: Waterlogged, Wheat, Endosperm, Anatomical structure, Transcriptome, Differentially expressed gene

\footnotetext{
* Correspondence: ouyangxq@163.com

${ }^{4}$ School of Life Science and Technology, Henan Institute of Science and Technology, Xinxiang 453003, China

Full list of author information is available at the end of the article
}

C C The Author(s). 2020 Open Access This article is licensed under a Creative Commons Attribution 4.0 International License, which permits use, sharing, adaptation, distribution and reproduction in any medium or format, as long as you give appropriate credit to the original author(s) and the source, provide a link to the Creative Commons licence, and indicate if changes were made. The images or other third party material in this article are included in the article's Creative Commons licence, unless indicated otherwise in a credit line to the material. If material is not included in the article's Creative Commons licence and your intended use is not permitted by statutory regulation or exceeds the permitted use, you will need to obtain permission directly from the copyright holder. To view a copy of this licence, visit http://creativecommons.org/licenses/by/4.0/ The Creative Commons Public Domain Dedication waiver (http://creativecommons.org/publicdomain/zero/1.0/) applies to the data made available in this article, unless otherwise stated in a credit line to the data. 


\section{Background}

Due to high rainfall, irrigation practices and/or poor soil drainage, waterlogging annually affects large areas of farmlands worldwide, and these effects result in anoxic (absence of $\mathrm{O}_{2}$ ) soils and severe hypoxia or anoxia within crop roots. Hypoxia caused by waterlogging can inhibit the growth of crop roots and stems, the accumulation of dry matter and the final yield [1]. Oxygen is crucial for generation of the ATP needed to drive secondary energy-dependent ion transport. The reduced uptake of nutrients under hypoxic conditions is mainly due to inefficient oxygen transport down to the root [2]. Relevant studies have shown that waterlogging might affect the hormone content in wheat roots and stems, possibly by impacting the production of ethylene [3], and then reduce the absorption of mineral nutrients by plant roots [4]. Wheat is an important food crop widely planted throughout the world and is ranked first in terms of harvest area among the three major food crops (rice, wheat and corn). China is the largest wheatproducing and wheat-consuming country in the world, and wheat is of great significance to China's food security and Chinese farmers' incomes. According to the World Food and Agriculture Organization (FAO), approximately $10 \%$ of the world's land area is affected by waterlogging to varying degrees [5]. In the Mediterranean region, the germination and growth stages of winter wheat are vulnerable to waterlogging because approximately $40 \%$ of the annual rainfall occurs during the sowing period of winter wheat [6]. In addition, the uneven terrain or poor drainage systems of farmlands easily cause waterlogging in the soil, which often leads to a lack of oxygen supply to the soil and the inhibition of seed germination, and these effects reduce the germination rate (GR) of wheat [7] and result in reductions in wheat production.

Both the timing and duration of waterlogging affect crop yields. In the case of wheat, the two stages at which waterlogging is most detrimental to wheat yields after germination and emergence are the seedling stage and the flowering stage [8]. Setter and Waters [8] found that waterlogging was most damaging during pre-emergence because it mostly results in the killing of seeds and/or very young seedlings. Water near the soil surface floods all the tissues of wheat seeds/seedlings, including the coleoptiles $[9,10]$. In contrast to rice, wheat seeds cannot germinate under anoxic conditions. Specifically, wheat seeds under hypoxia cannot decompose starch into sugar and thus cannot germinate. The supply of exogenous sugar results in germination of $84 \%$ of seeds, but only slight root elongation (a few millimetres) and no coleoptile growth have been observed in the absence of hypoxia [11]. Moreover, short waterlogging periods of 1-3 days can result in long-term detrimental effects on both the growth and yield of wheat [12]. Researchers believe that plants have established a set of new anatomical, morphological and physiological mechanisms to adapt to waterlogging environments [10], and these mechanisms include the production of aerenchyma, the formation of adventitious roots, petiole elongation, the growth of stem hypertrophy, the growth of hypocotyls, increases in plant height, stomatal closure, the reduction of transpiration and the inhibition of photosynthesis [2, $4,13]$. In the wheat research field, more attention has been paid to the response of plants to waterlogging at the seedling and filling stages [3, 14], but few studies have investigated the response of wheat to waterlogging stress at the germination stage.

Transcriptome sequencing technology can identify and clarify the metabolite synthesis pathways and can accelerate research on plant metabolism. RNA-Seq technology has been used to analyse the differential expression profile of waterlogging-tolerant and waterloggingsensitive rice varieties during germination to rapidly screen several important candidate genes, such as OsTPP7, OsHXK7 and OsPGM, which might affect the early stage of rice growth [15]. The germination of seeds is mainly dependent on amyloplasts in the endosperm [16]; therefore, we focused our analysis on the structural changes in the endosperm at the seed germination stage. In this study, the phenotype and anatomical structures of different wheat genotypes (waterlogging-tolerant, waterlogging-medium tolerant, and waterloggingsensitive varieties) during seed germination were analysed, and the transcriptional profile during germination was then analysed. Comparisons of the structural and transcriptional profiles of three types of wheat under the waterlogging and control treatments revealed several key DEGs involved in important metabolic pathways under the waterlogging treatment. This study establishes a foundation for further study on the functional characteristics of key waterlogging resistance genes in wheat and is of great significance for the genetic improvement of waterlogging-resistant wheat varieties.

\section{Results}

Phenotypic analysis of different wheat varieties under the waterlogging treatment

The phenotypes of three wheat seeds (ZM22, BN207 and BN607) after 3 days of germination under the waterlogging treatment are shown in Fig. 1. Under the control treatment, no significant difference in the coleoptile height $(\mathrm{CH})$ was found among the three wheat varieties (Fig. 1a). The GR and $\mathrm{CH}$ of the three types of wheat were higher than $95 \%$ and approximately $3.5 \mathrm{~cm}$, respectively (Fig. 1b and d). Although no significant differences were found among the roots of ZM22, BN207 and $\mathrm{BN607}$, the $\mathrm{GR}$ and $\mathrm{CH}$ of the three wheat varieties 


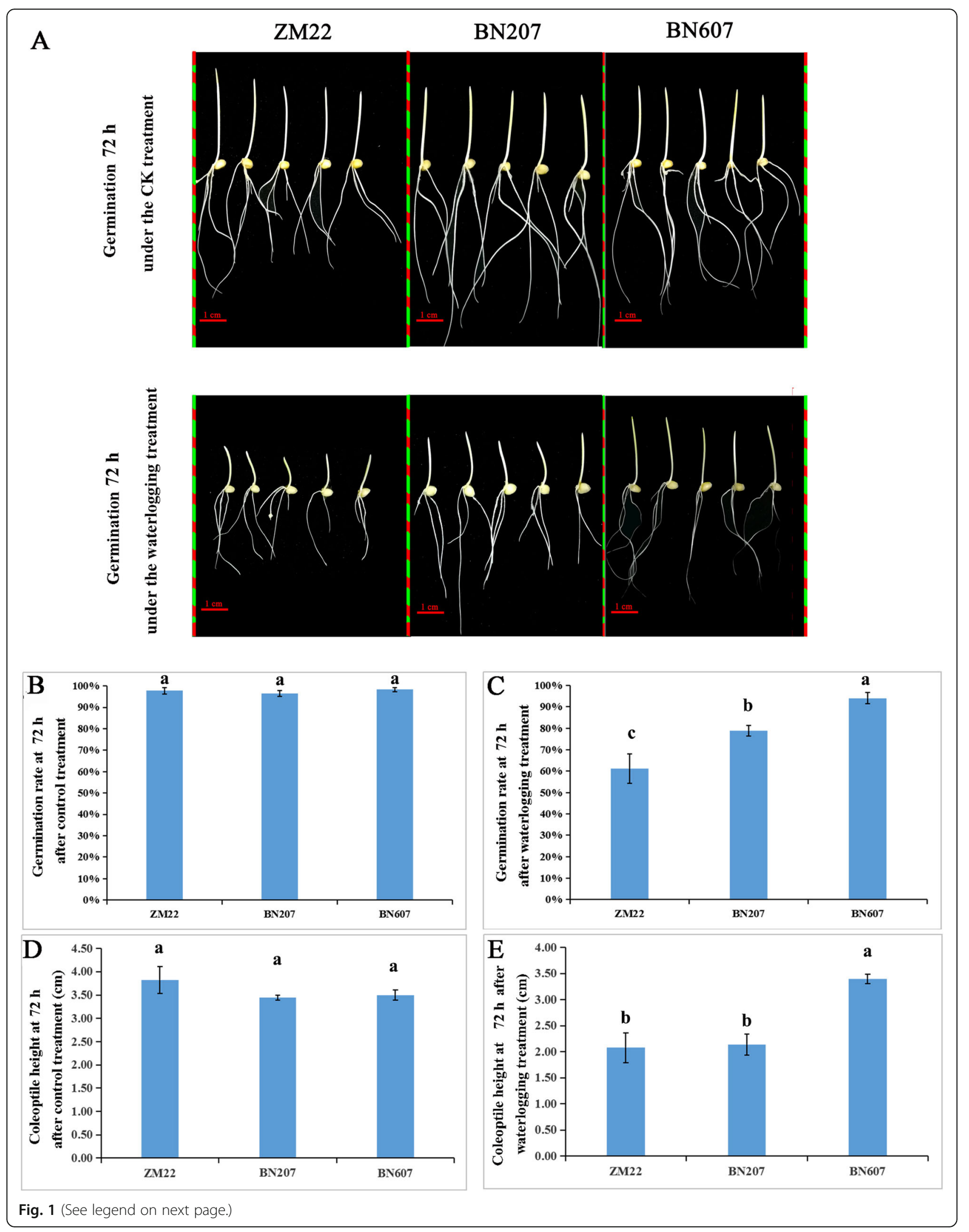


(See figure on previous page.)

Fig. 1 Phenotypic analysis of different wheat varieties after $72 \mathrm{~h}$ of germination under the control and waterlogging treatments. a, Phenotypes of ZM22, BN207 and BN607 seeds after $72 \mathrm{~h}$ of germination under the control and waterlogging treatments. Bars: $1 \mathrm{~cm}$. $\mathbf{b}$, Germination rate of ZM22, BN207 and BN607 after $72 \mathrm{~h}$ of the control treatment. c, Germination rate of ZM22, BN207 and BN607 after $72 \mathrm{~h}$ of the waterlogging treatment. d, Coleoptile height of ZM22, BN207 and BN607 after $72 \mathrm{~h}$ of the control treatment. e, Coleoptile height of ZM22, BN207 and BN607 after $72 \mathrm{~h}$ of the waterlogging treatment. Each point represents the average from five samples. The error bars represent the SDs. The statistical analysis was performed using the LSD test with $p<0.05$

under the waterlogging treatment were significantly different from those under the control treatment. The highest GR was found with BN607 (94.02\%), followed by BN207 (78.85\%), and the lowest GR was obtained with ZM22 (61.21\%) (Fig. 1c and e). In addition, the CHs of ZM22 and BN207 under the waterlogging treatment (average height of approximately $2 \mathrm{~cm}$ ) were significantly lower than those under the control treatment, whereas that of BN607 under the waterlogging treatment was equal to $3.5 \mathrm{~cm}$ and was not significantly different compared that under the control treatment (Fig. 1e). Based on the above analysis, we ranked the three wheat varieties based on their waterlogging tolerance levels of (from high to low) as BN607, BN207 and ZM22.

\section{Anatomical analysis of different wheat seeds under the waterlogging treatment}

The structural anatomical analysis of the seeds showed that the endosperm was emulsified and degraded during seed germination. The endosperm began to emulsify at the area adjacent to the embryo, which resulted in the formation of a cavity (Fig. 2a). The emulsification degrees of the endosperms of the three wheat varieties after $72 \mathrm{~h}$ of waterlogging treatment were different. The highest degree of endosperm emulsification was obtained for BN607, followed by BN207 and ZM22 (Fig. 2a). As demonstrated by scanning slices of the three wheat varieties, no significant difference in the starch grain size of ZM22 between the waterlogging and control treatments, but the number of amyloplasts under the waterlogging treatment was significantly lower than that under the control treatment (Fig. 2b). In BN607, the length and width of the amyloplasts under the waterlogging treatment were lower than those under the control treatment, and the number of amyloplasts under the waterlogging treatment was significantly lower than that under the control treatment (Fig. 2c). In BN207, no significant difference in the starch size was detected between the waterlogging and control treatments. Surprisingly, the number of amyloplasts in ZM22 under the waterlogging treatment was significantly higher than that under the control treatment (Fig. 2c). We concluded that the amyloplasts of ZM22, BN207 and BN607 might exhibit varying levels of responses to waterlogging stress.
Transcriptomic analysis of three wheat varieties under the waterlogging and control treatments

To study the response mechanism of wheat seeds to waterlogging stress during germination, samples were collected from BN607, BN207 and ZM22 under the waterlogging and control treatments. Each variety was subjected to three replicate treatments, and a total of 18 libraries were constructed. We subsequently performed transcriptome sequencing using Illumina NovaSeq ${ }^{\mathrm{Tm}} 6000$ with the wheat genome was used as the reference genome, and an average of 6.40 million reads were obtained from each sample. After removing low-mass, joint, and potentially contaminated data, 7.41-10.38 GB data were obtained from each sample, the GC value was 53.00$54.00 \%$, and the Q30 value ranged from 97.62 to $98.44 \%$ (Table S1). The ratio of exons to reference genomic libraries averaged $92.35 \%$, whereas the average ratios obtained for introns and intergenic spacers were 2.67 and $4.98 \%$, respectively (Table S2).

A principal component analysis (PCA) of all the genes in the 18 libraries (Fig. S1) revealed that principal component 1 (PC1) and PC2 explained 79.09 and $11.85 \%$ of the variance. The three biological replicates of each treatment were significantly clustered together, and similar results were obtained with the waterlogging-treated and normally cultured wheat varieties. These results showed that the biological replicates of the samples were good and that the sequencing results were reliable.

\section{Analysis of DEGs in three wheat varieties in response to waterlogging stress}

A total of 107,891 genes were obtained from the transcriptome sequencing data, and 479, 637 and 1800 DEGs were identified in ZM22, BN207 and BN607 under waterlogging stress, respectively (Fig. 3a). As determined through a GO enrichment analysis of these DEGs (Fig. S2, Table S3), the DEGs in ZM22 mostly functioned in oxidoreductase activity and mainly participated in the biological response to abscisic acid. In addition, the DEGs in BN207 mainly functioned in molecular function and DNA binding, mostly exhibited protein heterodimerization activity and mainly participated in the biological response to abscisic acid, the response to water deprivation and the defence response to fungus. Moreover, the DEGs in BN607 mostly showed transcription factor activity 


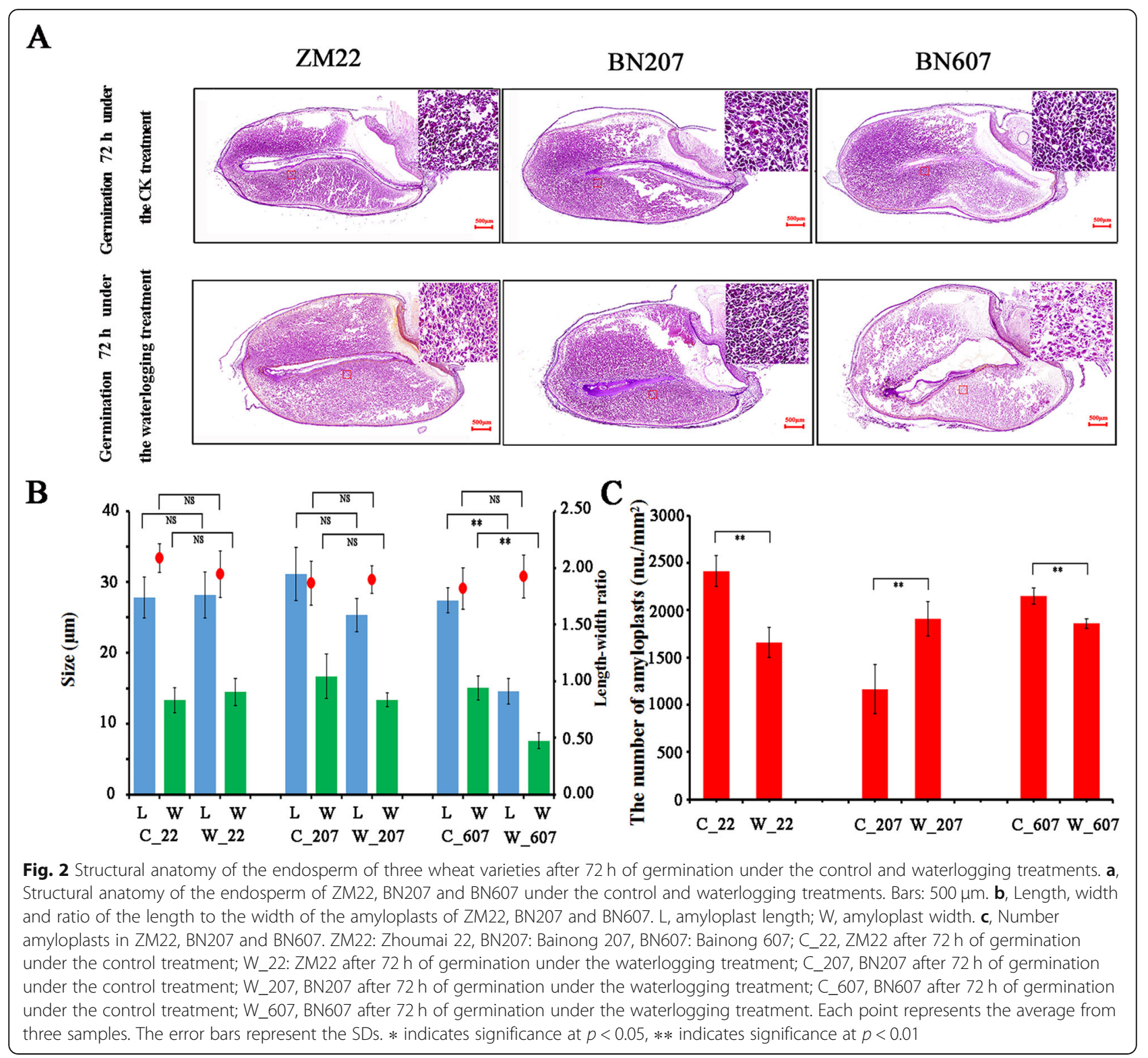

and mainly participated in the biological oxidationreduction process. A KEGG enrichment analysis showed that 13 DEGs in ZM22 were significantly enriched in the "amino sugar and nucleotide sugar metabolism" pathway (Fig. S3, Table S4), followed by the "glyceride metabolism" and "base sugar and nucleotide sugar metabolism" pathways. In BN607 under waterlogging stress, 40 genes were significantly enriched in the "phenylpropanoid biosynthesis" and "starch and sucrose metabolism" pathways followed by the "amino sugar and nucleotide sugar metabolism" pathway.

A total of 2775 genes were significantly expressed in three wheat varieties under waterlogging stress. In ZM22, 378 genes were significantly downregulated, and 103 genes were significantly upregulated (Fig. 3a), and 302 and 335 genes were significantly downregulated and significantly upregulated in BN207, respectively (Fig. 3a). In BN607, 1116 and 684 genes exhibited significantly downregulated expression and significantly upregulated expression, respectively (Fig. 3a). A Venn diagram of 2775 DEGs showed that 1677 DEGs were uniquely found in BN607, and 1054 and 623 genes were downregulated and upregulated, respectively. In addition, five DEGs were shared among the three varieties. The expression difference levels of four genes (Traes $C$ SU02G032900, MSTRG.18850, TraesCS6D02G038500 and TraesCS1A02G139900) were downregulated in the three varieties under waterlogging stress, and the expression level of TraesCS5B02G445500 was upregulated under waterlogging stress (Fig. 3b, Table S5), which suggested that these 


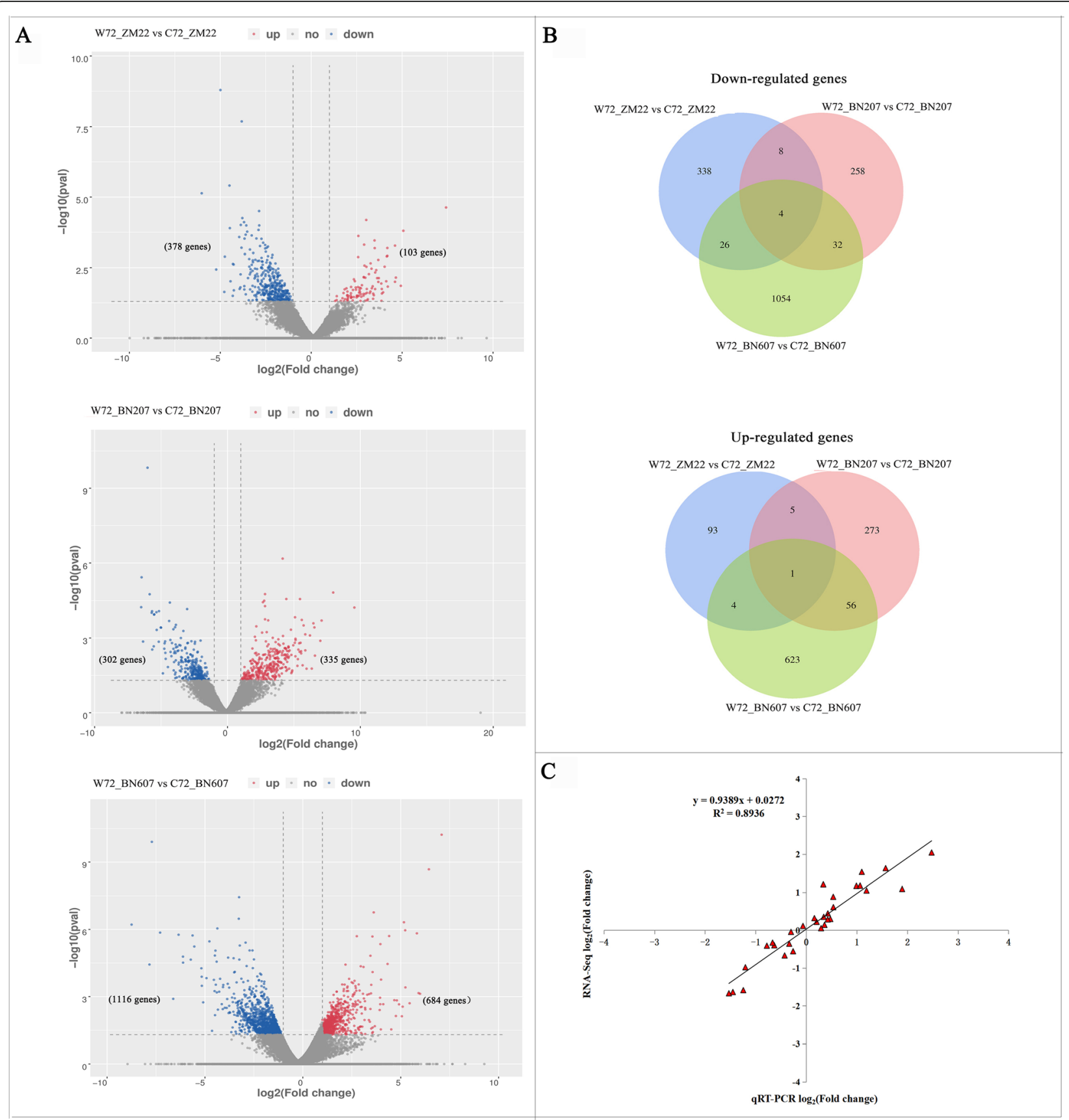

Fig. 3 Analysis of DEGs in three wheat cultivars under waterlogging stress. a. Volcano plot analysis of DEGs in ZM22, BN206 and BN607. The abscissa represents the $\log _{2}$ (fold change), and $-\log _{2}$ ( $p$-value) is shown as the ordinate. The red colour indicates significantly upregulated DEGs, the blue colour showed the significantly downregulated DEGs, and the grey colour represents nonsignificant DEGs. $\mathbf{b}$. Venn diagram of downregulated and upregulated genes in ZM22, BN206 and BN607. c. Correlation analysis of the qRT-PCR data and the RNA sequencing results. W72_ZM22, W72_BN207 and W72_BN607 refer to ZM22, BN207 and BN607 under the waterlogging treatment, respectively. C72_ZM22, C72_BN207 and C72_BN607 refer to ZM22, BN207 and BN607 under the control treatment, respectively

genes might play an important role in three wheat varieties under waterlogging stress.

To validate the reliability of the expression profiles obtained by RNA-Seq, we randomly selected 10 DEGs that showed various expression levels for validation by quantitative real-time polymerase chain reaction (qRT-PCR). Pearson's correlation coefficients showed that the qRT-PCR and RNA sequencing data for these genes were highly correlated (Fig. 3c). The correlation coefficient was 0.8936 , which indicated a 
positive correlation between the RNA sequencing and qRT-PCR data (Fig. 3c).

\section{Selection of gene set response to waterlogging stress by hierarchical clustering based on DEGs}

According to the differences in the expression of the DEGs among the three waterlogging-resistant varieties (BN607 > BN207 > ZM22), the DEGs were stratified and clustered (Fig. 4a). The results showed that these DEGs were divided into eight clusters with different expression patterns (clusters 1 to 8, Fig. 4b, Table S7). Among these clusters, cluster 7 contained 563 DEGs, and the expression of these DEGs in the three varieties was positively correlated with the GR $\left(R^{2}=1.00\right)$. In contrast, cluster 2 contained 398 DEGs, and the expression of these genes in the three varieties was negatively correlated with the GR $\left(R^{2}=-0.97\right.$, Fig. 4c). The results from the above analysis suggested that the DEGs in clusters 2 and 7 play important roles in the response of the three wheat cultivars to waterlogging stress.

\section{Main metabolic pathways involved in wheat germination under waterlogging stress}

Based on the hierarchical clustering analysis of the DEGs, we focused on analysing the DEGs in clusters 2 and 7 that are involved in waterlogging stress. GO and KEGG enrichment analyses revealed that these genes were mainly involved in the glycolysis pathway, the starch and sucrose metabolism pathway and the lactose metabolism pathway.

\section{Analysis of DEGs related to the glycolytic pathway}

The KEGG enrichment analysis showed that many DEGs in the three types of wheat under the waterlogging and control treatments were involved in the glycolysis pathway (Fig. 5a). We screened four DEGs in clusters 2 and

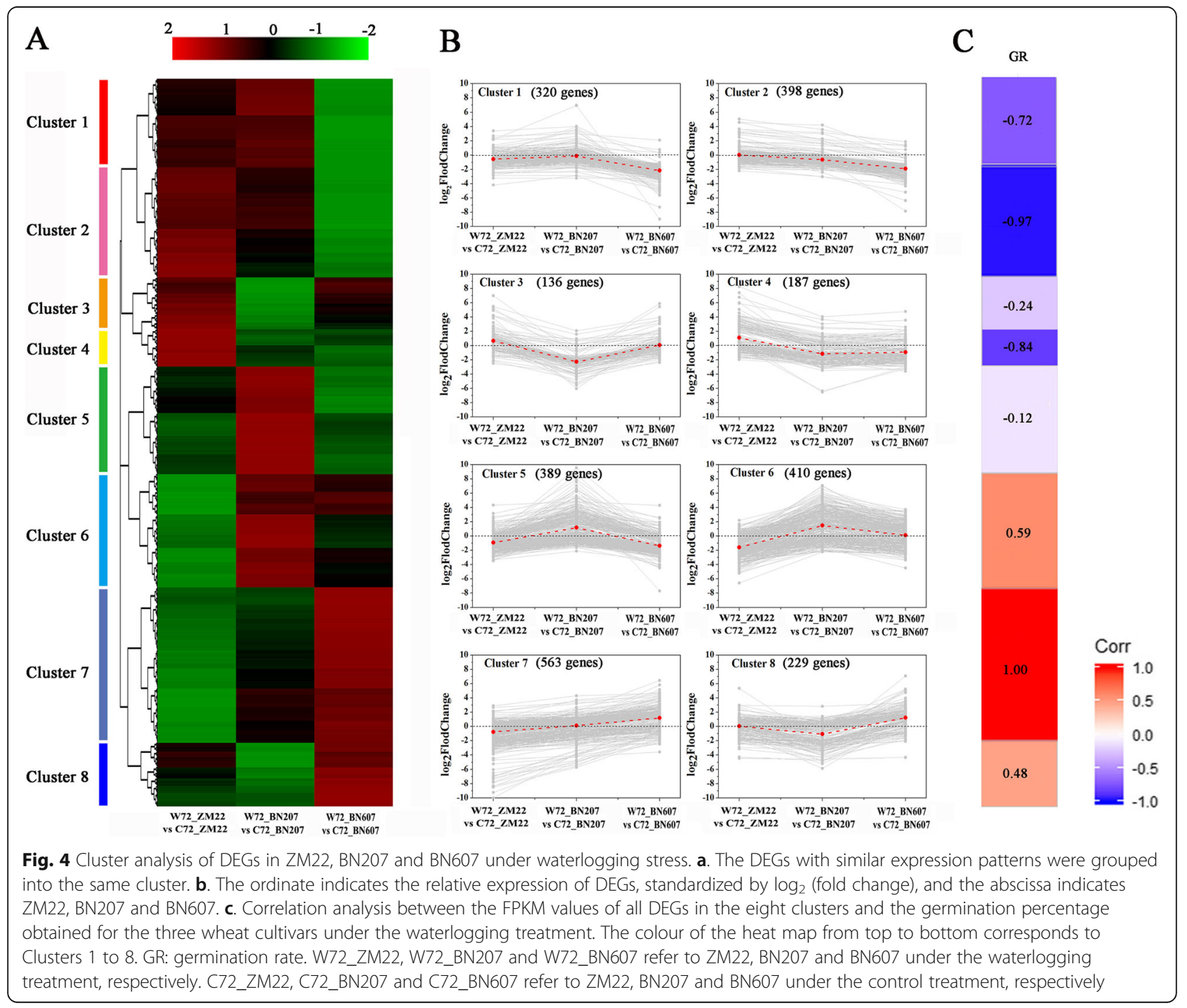




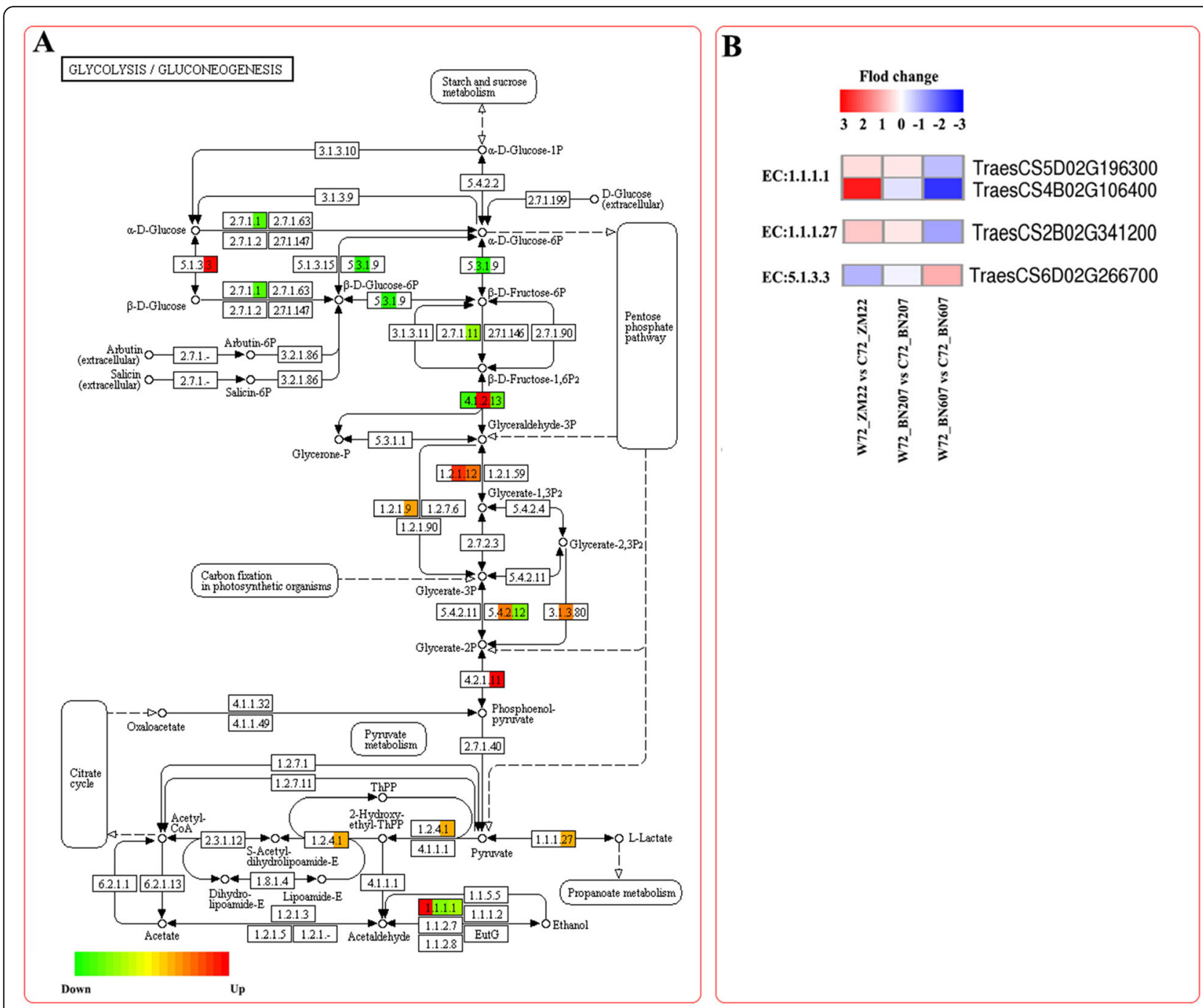

Fig. 5 Analysis of DEGs involved in the glycolysis pathway in ZM22, BN207 and BN607. a, Pattern diagram of the glycolysis pathway. The red squares indicate upregulated genes, the green squares indicate downregulated genes, and the orange squares indicate both upregulated and downregulated genes. $\mathbf{b}$, The expression difference levels of DEGs involved in the glycolysis pathway between waterlogging treatment and control treatment in three varieties. The metabolism of each previously selected DEGs was analysed using a heatmap. Relative expression level of DEGs was measured by log2 ratio (Fold change between waterlogging treatment and control treatment each variety). Positive fold change values (red) indicate the up-regulation, whereas negative fold change values (blue)

7 (two ADH genes, one L-lactate dehydrogenase gene, and one glycosyltransferase gene) that were closely related to the GR (Fig. 5b). The expression difference levels of two genes (TraesCS5D02G196300 and TraesCS4B02G106400) encoding alcohol dehydrogenase decreased successively in ZM22, BN206 and BN607 (Fig. $5 \mathrm{~b})$. In addition, the expression trend of the L-lactate dehydrogenase gene (TraesCS2B02G341200), which catalyses the conversion of pyruvate to L-lactate (EC: 1.1.1.27), was opposite to that found for the $\mathrm{ADH}$ gene in the three wheat varieties. Glycosyltransferase catalyses the conversion of $\alpha$-D-glucose into $\beta$-D-glucose (EC: 5.1.3.3), and the expression difference levels of the gene
(TraesCS2B02G341200) encoding this function decreased successively in ZM22, BN206 and BN607.

\section{Analysis of DEGs related to the starch and sucrose metabolism pathway}

The KEGG enrichment revealed that a large number of the DEGs in the three wheat varieties under the waterlogging and control treatments were involved in the starch and sucrose metabolism pathway (Fig. 6a), and 18 of the DEGs in clusters 2 and 7 that were involved in the starch and sucrose metabolism pathway were screened (Fig. 6b). A gene (TraesCS5B02G015800) belonging to cluster 7 was annotated as alpha-amylase, and 

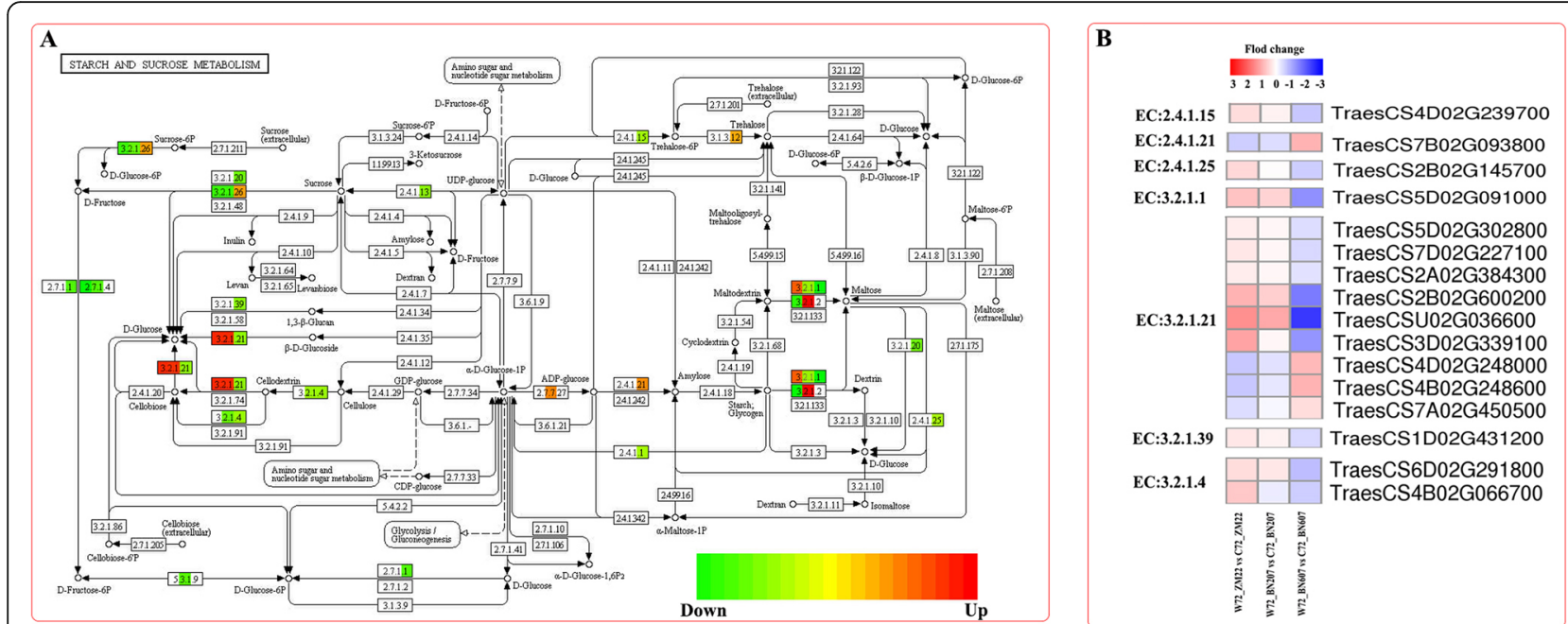

Fig. 6 Analysis of DEGs involved in the starch and sucrose metabolic pathway in ZM22, BN207 and BN607. a, Pattern diagram of the starch and sucrose metabolic pathway. The red squares indicate upregulated genes, the green squares indicate downregulated genes, and the orange squares indicate both upregulated and downregulated genes. b. The expression difference levels of DEGs involved in the starch and sucrose metabolic pathway between waterlogging treatment and control treatment in three varieties. The metabolism of each previously selected DEGs was analysed using a heatmap. Relative expression level of DEGs was measured by log2 ratio (Fold change between waterlogging treatment and control treatment each variety). Positive fold change values (red) indicate the up-regulation, whereas negative fold change values (blue)

the expression difference levels increased successively in ZM22, BN206 and BN607, which indicated that this gene might play an important role in starch decomposition. We screened the gene (TraesCS7B02G093800) encoding maltitol glycosylase (EC: 2.4.1.21), and these expression difference levels successively increased in ZM22, BN206 and BN607. In addition, the expression difference levels of TraesCS5D02G09100, which encodes the enzyme that catalyses the conversion of starch/glycogen to dextrin, was decreased successively in ZM22, BN206 and BN607. This result indicated that the ability of ADP-glucose to control amylose synthesis was gradually enhanced. Chitinase was used to catalyse the decomposition of $\beta$-D-glucose or fibre to D-glucose (EC: 3.2.1.21). The expression difference levels of six genes encoding this function decreased gradually in ZM22, BN206 and BN607, but the opposite trend was found for three other genes (Fig. 6b). In this study, the expression difference levels of the genes encoding glucan-1,3-glucosidase (EC: 3.2 .1 .39 ) and endocellulase (EC: 3.2 .1 .4$)$ decreased gradually in ZM22, BN206 and BN607 (Fig. 6b), which indicated that these genes might play an important role in the waterlogging tolerance of different varieties.

\section{Analysis of DEGs related to the lactose metabolic pathway}

A comprehensive analysis of the DEGs in the three wheat varieties belonging to clusters 2 and 7 and involved in the lactose metabolism pathway of (Fig. 7a) identified two genes (TraesCS1A02G266600 and
TraesCS1D02G266700) that encode glucuronosyltransferase (inositol galactoside synthase) (EC: 2.4.1.123). This protein catalyses the synthesis of inositol galactoside from UDP-galactose and myoinositol, which is the first key step in the synthesis of raffinose family oligosaccharides (RFOs) and the most critical regulatory step in RFO synthesis. The expression difference levels of these two genes were decreased successively in ZM22, BN206 and BN607. The analysis identified three genes encoding inositol galactoside-sucrose galactosyltransferase (raffinose synthase) (EC: 2.4.1.82). This protein mainly catalyses the synthesis of raffinose from inositol galactoside and sucrose. The expression difference levels of the TraesCS7D02G236700 gene were gradually decreased in ZM22, BN206 and BN607, whereas those of the TraesCS7B02G035200 and TraesCS7D02G133500 genes were gradually increased in ZM22, BN206 and BN607. One gene (TraesCS5B02G557400) encoding $\beta$-D-fructofuranoside (EC:3.2.1.26), which mainly catalyses the decomposition of stachyose and raffinose into melibiose and the decomposition of sucrose into glucose and fructose, was identified. The expression difference levels of TraesCS5B02G557400 were gradually increased in ZM22, BN206 and BN607. $\beta$-galactosidase (EC:3.2.1.23) mainly catalyses the decomposition of lactose and raffinose into melibiose. We also found that the expression difference levels of two genes (TraesCS7A02G0363600 and TraesCS1B02G278400) gradually increased in ZM22, BN206 and BN607. 


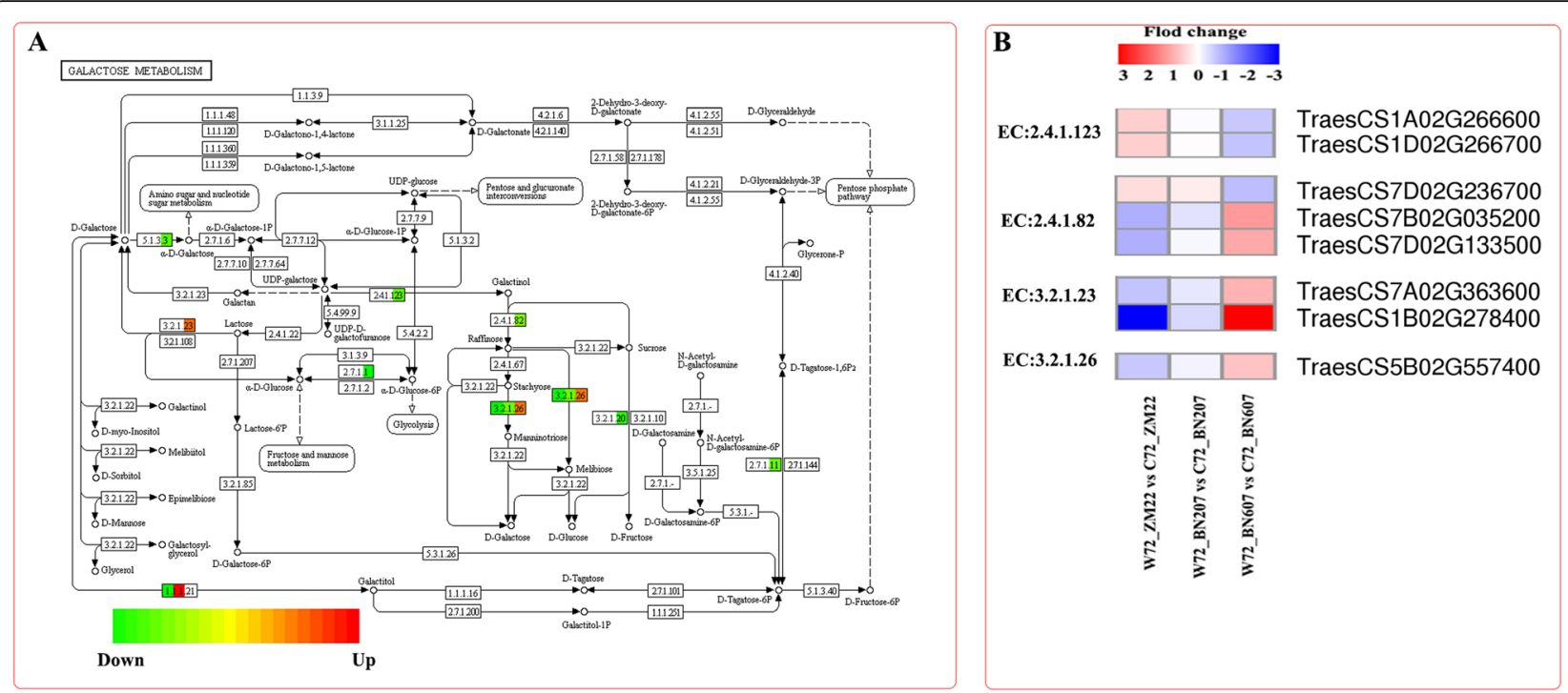

Fig. 7 Analysis of DEGs involved in the lactose metabolic pathway in ZM22, BN207 and BN607. a, Pattern diagram of the lactose metabolic pathway. The red squares indicate upregulated genes, the green squares indicate downregulated genes, and the orange squares indicate both upregulated and downregulated genes. b. The expression difference levels of DEGs involved in the lactose metabolic pathway between waterlogging treatment and control treatment in three varieties. The metabolism of each previously selected DEG was analysed using a heatmap. Relative expression level of DEGs was measured by log2 ratio (Fold change between waterlogging treatment and control treatment each variety). Positive fold change values (red) indicate the up-regulation, whereas negative fold change values (blue)

\section{Analysis of DEGs related to transcription factors}

The analysis of the DEGs in clusters 2 and 7 revealed 23 transcription factors involved in waterlogging stress (Table 1). These transcription factors were distributed in 12 families, including the WRKY, MYB, MADS, ZIP, and ERF families (Table 1). Among the WRKY family, the expression difference levels of five genes was highest in ZM22, followed by BN207 and then BN607, whereas the expression difference level of one gene was lowest in ZM22, followed by BN207 and then BN607. A total of four genes related to the MYB family were found: The expression difference levels of one of these genes gradually decreased in ZM22, BN206 and BN607, and those of the other three genes gradually increased. In addition, the expression difference levels of MSTRG.6000, TraesCS1B02G392300 and MSTRG.32294, which are all ethylene-related transcription factors (ERF, AP2/ERF, and $A B R$ ) belonging to cluster 7 , gradually increased in ZM22, BN206 and BN607. This finding indicated that these transcription factors might play an important role in the response to waterlogging stress in different wheat varieties.

\section{Discussion}

\section{Response of the GR of different wheat varieties to waterlogging stress}

Waterlogging often occurs at all stages of plant growth; therefore, evaluating the waterlogging tolerance of plants at each stage is highly important. Studies of the physiological and molecular mechanisms related to the effects of waterlogging in wheat at the seedling stage [9, 14], filling stage [17] and maturity stage [18] have been reported. Some studies have revealed that wheat, barley and rape are markedly more likely to suffer from waterlogging within 2 and 6 weeks after germination than between 6 and 14 weeks after germination. In addition, Cannell et al. [19] found that after wheat germination, all seedlings died after 16 days of waterlogging at $12{ }^{\circ} \mathrm{C}$, whereas the survival rate of wheat seedlings after 6 days of waterlogging was $12-38 \%$ of that found with the control treatment. Considerable differences in waterlogging tolerance have been found among different varieties in different regions. Takeda and Fukuyama [20] found that some varieties from China, Japan, South Korea and Nepal, as well as some varieties from North Africa, Ethiopia and southwestern Asia, tend to exhibit higher "waterlogging tolerance", whereas barley varieties from western India show weaker "waterlogging tolerance" [8]. The plant height, soil and plant analyser development (SPAD) results, number of tillers, and biomasses of the stems and roots of non-waterlogging wheat varieties are lower than those of waterlogging-tolerant wheat varieties [21]. This study revealed that the GRs of ZM22, BN207 and BN607 increased gradually during waterlogging treatment. The $\mathrm{CH}$ and root length of ZM22 were seriously inhibited by waterlogging treatment for $72 \mathrm{~h}$, whereas the growth of BN607 was not affected by the waterlogging treatment (Figs. 1 and 3), which indicated 
Table 1 Response of transcription factors to waterlogging stress

\begin{tabular}{|c|c|c|c|c|c|c|c|}
\hline \multirow[t]{2}{*}{ Category } & \multirow[t]{2}{*}{ Gene_id } & \multirow[t]{2}{*}{ Cluster } & \multicolumn{3}{|c|}{$\underline{\log }_{2}$ (Fold change) } & \multirow{2}{*}{$\begin{array}{l}\text { Swissprot_ } \\
\text { name }\end{array}$} & \multirow[t]{2}{*}{ Swissprot_description } \\
\hline & & & $\begin{array}{l}\text { W72_ZM22 vs } \\
\text { C72_ZM22 }\end{array}$ & $\begin{array}{l}\text { W72_BN207 vs } \\
\text { C72_BN207 }\end{array}$ & $\begin{array}{l}\text { W72_BN607 vs } \\
\text { C72_BN607 }\end{array}$ & & \\
\hline \multirow[t]{6}{*}{$\overline{\text { WRKY }}$} & TraesCS1A02G197400 & $\mathrm{C} 2$ & -0.13 & -0.38 & -1.55 & WRKY35 & WRKY transcription factor 35 \\
\hline & TraesCS3A02G347500 & $\mathrm{C} 2$ & -0.4 & -0.57 & -1.83 & WRKY24 & $\begin{array}{l}\text { WRKY transcription factor } \\
\text { WRKY24 }\end{array}$ \\
\hline & TraesCS6A02G146900 & $\mathrm{C} 2$ & -0.99 & -1.07 & -2.16 & WRKY71 & $\begin{array}{l}\text { WRKY transcription factor } \\
\text { WRKY71 }\end{array}$ \\
\hline & TraesCS6B02G175100 & $\mathrm{C} 2$ & -0.98 & -1.4 & -2.14 & WRKY71 & $\begin{array}{l}\text { WRKY transcription factor } \\
\text { WRKY71 }\end{array}$ \\
\hline & TraesCS2B02G410400 & $\mathrm{C} 2$ & -0.13 & -0.61 & -1.51 & WRKY12 & WRKY transcription factor 12 \\
\hline & TraesCS3D02G113300 & $\mathrm{C7}$ & -2.2 & -0.49 & 0.15 & WRKY72 & WRKY transcription factor 72 \\
\hline \multirow[t]{4}{*}{ MYB } & TraesCS1D02G084400 & $\mathrm{C} 2$ & 0.04 & -0.65 & -1.68 & MYB86 & Transcription factor MYB86 \\
\hline & TraesCS7D02G514800 & $\mathrm{C7}$ & -3.69 & -1.34 & -0.03 & MYB44 & Transcription factor MYB44 \\
\hline & TraesCS2A02G157600 & $\mathrm{C7}$ & -1.46 & -0.46 & 0.19 & MYB306 & Myb-related protein 306 \\
\hline & TraesCS2A02G116100 & $\mathrm{C} 7$ & 0.7 & 0.78 & 1.03 & EFM & $\begin{array}{l}\text { Myb family transcription factor } \\
\text { EFM }\end{array}$ \\
\hline \multirow[t]{3}{*}{ ERF } & TraesCS2A02G288000 & C7 & -0.06 & 0.32 & 1.12 & ERF114 & $\begin{array}{l}\text { Ethylene-responsive transcription } \\
\text { factor ERF114 }\end{array}$ \\
\hline & TraesCS1B02G392300 & $\mathrm{C7}$ & -0.41 & 0.73 & 1.95 & AP2/ERF & $\begin{array}{l}\text { AP2/ERF and B3 domain-containing } \\
\text { protein }\end{array}$ \\
\hline & TraesCS7A02G264100 & $\mathrm{C7}$ & -0.2 & 0.39 & 1.08 & ABR1 & $\begin{array}{l}\text { Ethylene-responsive transcription } \\
\text { factor } A B R 1\end{array}$ \\
\hline \multirow[t]{2}{*}{ MADS-box } & TraesCS5B02G115100 & $\mathrm{C} 2$ & 0.17 & 0.06 & -2.07 & MADS13 & MADS-box transcription factor 13 \\
\hline & TraesCS4D02G346300 & $\mathrm{C7}$ & 0.07 & 0.96 & 1.16 & MADS51 & MADS-box transcription factor 51 \\
\hline \multirow[t]{2}{*}{ ZIP } & TraesCS2A02G424200 & $\mathrm{C} 2$ & 0.14 & -0.34 & -1.26 & ZIP3 & Zinc transporter 3 \\
\hline & TraesCS1B02G306500 & $\mathrm{C} 2$ & -0.14 & -0.34 & -1.36 & ZIP5 & Zinc transporter 5 \\
\hline \multirow[t]{2}{*}{$\mathrm{BHLH}$} & TraesCS2D02G347200 & $\mathrm{C} 7$ & -1.1 & -0.56 & 1.62 & BHLH113 & Transcription factor bHLH113 \\
\hline & TraesCS5D02G411600 & $\mathrm{C7}$ & -2.61 & 0.24 & 1.16 & BHLH84 & Transcription factor bHLH84 \\
\hline IAA & TraesCS7A02G322000 & $\mathrm{C} 2$ & -0.74 & -0.99 & -1.55 & IAA25 & Auxin-responsive protein IAA25 \\
\hline ARF & TraesCS3B02G190100 & $\mathrm{C} 2$ & -0.1 & -0.64 & -1.34 & ARF1 & Auxin response factor 1 \\
\hline HSFA & TraesCS2D02G211400 & $\mathrm{C7}$ & -1.72 & -0.81 & 0.53 & HSFA3 & Heat stress transcription factor $\mathrm{A}-3$ \\
\hline NAC & TraesCS2A02G102000 & $\mathrm{C7}$ & 0.24 & 0.69 & 1.24 & NAM-B1 & NAC transcription factor NAM-B1 \\
\hline
\end{tabular}

NOTE: C72_ZM22, ZM22 after $72 \mathrm{~h}$ of germination under the control treatment; W72_ZM22: ZM22 after $72 \mathrm{~h}$ of germination under the waterlogging treatment; C72_BN207, BN207 after $72 \mathrm{~h}$ of germination under the control treatment; W72_BN207, BN207 after $72 \mathrm{~h}$ of germination under the waterlogging treatment; C72_BN607, BN607 after $72 \mathrm{~h}$ of germination under the control treatment; and W72_BN607, BN607 after $72 \mathrm{~h}$ of germination under the waterlogging treatment

the existence of notable differences in the "waterlogging tolerance" among different varieties. The number of DEGs that were both up-regulated and down-regulated in the three varieties was very small (Fig. 3b). On the one hand, it may be related to the sampling period after the waterlogging treatment. This study only analyzed the period when the three varieties had significant differences in GR. For samples, studies have shown that significant changes have taken place inside the seeds when the seeds have been submerged for a few hours [8]. On the other hand, the speed of the physical and chemical changes in the embryo and endosperm of the three varieties of wheat seeds after waterlogging treatment is not consistent. Therefore, when sampling at a certain time point, there will be a phenomenon that the number of genes that were both up-regulated and down-regulated was small. However, it could be observed in Fig. 4 that there were many DEGs in the three varieties after waterlogging treatment. The focus of this study is to screen for DEGs that have a significant positive and negative correlation with GR. The expression of DEGs respond to waterlogging treatments had a significant effect on GR, which provides us with an important resource to explain the different mechanisms of submergence tolerance of different wheat varieties and to study key candidate genes that regulate seed submergence tolerance during wheat germination. 


\section{Anatomy of the grain endosperm and endosperm in the response of wheat to waterlogging stress}

The endosperm is the main location of nutrient storage in cereal seeds and mainly contains starch. After degradation by amylase, starch is transported to the germ and radicle in the form of simple compounds (such as sucrose), provides a matrix for respiration and serves as a material basis and energy source for radicle and germ growth and organ formation [16, 22, 23]. At the initial stage of wheat seed germination, the cell wall of endosperm cells is dissolved, and the endosperm cells are separated. The starch and cellulose gradually decompose under a series of enzymes ( $\alpha-/ \beta$-amylase and cellulases), which loosens the entire endosperm structure and leads to endosperm liquefaction. Therefore, the activity of amylase in seed germination might be determined by the degrees of endosperm emulsification and degradation. This study revealed that the endosperm cells of three types of wheat seeds were markedly changed in response to waterlogging stress. Specifically, the degree of emulsification between the embryo and the endosperm found for BN207 and BN607 was higher than that obtained for ZM22, and BN607 exhibited the highest degree of emulsification (Fig. $2 b$ and $c$ ). The differences in the number and size of amyloplasts among ZM22, BN2607 and BN607 might be due to the inconsistencies in the degradation rate. For example, compared with the control treatment, the size of amyloplasts in ZM22 remained unchanged, but the number of amyloplasts was decreased by the waterlogging treatment. In addition, the size of amyloplasts in BN207 was decreased under the waterlogging treatment, but the difference between the control and waterlogging treatments was not significantly. Moreover, the number of amyloplasts in BN207 increased, and starch degradation was rapidly completed, which provided nutrients for embryo growth.

\section{Importance of the ADH gene in the response of wheat to waterlogging stress at the germination stage}

Seed germination requires an energy supply, and energy metabolism is activated only when the seed is imbibed. Studies have revealed that some proteins related to energy metabolism are phosphorylated in wheat embryos. For example, the upregulation of the expression of the $A D H$ gene under waterlogging stress servers as a positive signal. Alcohol fermentation in plants is activated under low oxygen stress conditions: pyruvate decarboxylase (PDC) first converts pyruvate to acetaldehyde, and alcohol dehydrogenase then convert acetaldehyde to ethanol. It has been reported that at the early stage of soybean growth and development, the expression of $A D H$ and $P D C$ in soybean roots is significantly upregulated under waterlogging stress [24-26]. It has also been found that hypoxia stress can lead to the production of fermentation alcohol products [PDC, lactate dehydrogenase (LDH), and ADH] in Arabidopsis thaliana [27]. Komatsu et al. [28] reported that there are at least six $A D H$ genes involved in the response of soybean to waterlogging stress, and one of these genes, $G m A D H 2$, is specifically expressed in soybean root tissue. Similarly, two $A D H$ genes have been cloned in cotton, and only one of these genes is expressed under waterlogging stress [29]. Zhang et al. [30] found that two of the three $A D H$ genes in kiwifruit were significantly upregulated in the roots after waterlogging treatment. Tougou et al. [26] found that the inhibitory effect of waterlogging stress on the growth of soybean seedlings was decreased in $G m A D H 2 I$-overexpressing transgenic soybeans, whereas the expression and activity of ADH in the transgenic soybeans were higher than those in the control soybeans, which suggested that the exposure of GmADH2 transgenic soybeans to waterlogging stress might induce changes in glycolysis and alcohol fermentation and improve the GR. The transcriptional levels of metabolites in the embryo and endosperm of rice seeds are increased by anaerobic treatment for $12 \mathrm{~h}$, and the greatest change in a large number of metabolites has been observed after $48 \mathrm{~h}$ of treatment [31]. This finding shows that the enzyme activity-catalysed energy metabolism is induced or activated at the beginning of germination, and the late changes in metabolites might be driven by transcription and translation because these occurred after the observed changes in the transcriptional abundance. In this study, we found that the transcriptional levels of $A D H 2$ (TraesCS5D02G196300) and $A D H 3$ (TraesCS4B02G106400) (Fig. 7) after $72 \mathrm{~h}$ of the waterlogging treatment were lower than those obtained with the control treatment, and the greatest decrease was observed in ZM22, followed by BN207 and then BN607 (data not shown). In contrast, the transcriptional levels of these genes increased gradually after 1 day of the waterlogging treatment (data not shown). This finding suggested that the $A D H$ genes in the endosperm of BN607 were induced rapidly after short-term waterlogging, and the glycolysis pathway provided the energy needed to induce the early germination of $\mathrm{BN607}$ seeds. However, the $A D H$ genes of ZM22 were highly expressed after $72 \mathrm{~h}$ of the waterlogging treatment. It is possible that the accumulation of toxic substances, such as acetaldehyde, at the early stage inhibits seed germination.

\section{Waterlogging affects sugar metabolism of endosperm and endosperm in wheat}

The energy metabolism pathway is not only related to the alcohol dehydrogenase-catalysed step of glycolysis but also associated with starch and sucrose metabolism and the lactose metabolism pathway $[32,33]$. We found 
that after $72 \mathrm{~h}$ of waterlogging treatment, the expression levels of genes related to glycolysis, starch and sucrose metabolism and lactose metabolism in the embryo and endosperm of BN607 seeds were lower than those in ZM22 seeds. Among these genes, the expression difference levels of genes related to hydrolytic enzymes, such as chitinase, glucan-1,3-glucosidase and endocellulase, decreased successively in ZM22, BN206 and BN607. The trend underlying the changes in these genes was largely consistent with that found for $A D H$ gene expression. The anatomical structure of the three wheat species and the expression levels of the analysed genes indicated that the emulsification and dissolution of the endosperm of BN607 seeds began early after waterlogging treatment, whereas the emulsification and dissolution of the embryo and endosperm of ZM22 seeds occurred relatively late. The transcriptional expression of mRNAs in ZM22 seeds was inhibited by waterlogging stress, and this inhibition occurred too late for the plant to switch to using starch and sugar hydrolysis to provide the energy needed for coleoptile growth.

The coleoptile is the protective tissue of young cotyledons of crops. The length and elongation rate of the coleoptile affect the early growth of crops. Coleoptile growth is considered the extension growth of coleoptile cells, and the power for this extension growth originates from continuous swelling and pressure. The extended growth of cells is limited by the cell wall; therefore, relaxation of the cell wall is necessary during coleoptile growth [34]. The relaxation of the cell wall is related to the degradation of hemicellulose, and a single hydrolase, such as $\beta$-glucanase, expansin, $\alpha$-amylase and $\beta$ galactosidase, can induce cell wall relaxation. The pectin polysaccharides in the cell wall are hydrolysed by $\beta$ galactosidase, and this process is positively correlated with cell wall relaxation [35]. This study also found that the $\mathrm{CH}$ of ZM22, BN207 and BN607 and the expression levels of the two genes encoding $\beta$-galactosidase were increased by $72 \mathrm{~h}$ of the waterlogging treatment (Fig. 7b). We also found that the expression difference levels of a gene encoding $\alpha$-amylase in cluster 7 also increased successively in ZM22, BN206 and BN607. This finding showed that the elongation of the wheat coleoptile under waterlogging stress might be closely related to the changes in the cell wall composition and the synthesis and activity of induced enzymes related to cell wall relaxation.

\section{Role of transcription factors in the response to waterlogging stress}

Previous studies have found that WRKY-type genes play an important role in the responses to external abiotic stresses, such as drought, high salinity, cold injury, and heat damage [36], and some studies have also investigated WRKY-type genes in response to waterlogging stress. Nanjo et al. [25] found that the expression levels of WRKY-type transcription factors in soybean roots and hypocotyls are significantly upregulated under waterlogging treatment, and the expression levels of 11 WRKY transcription factor genes in the roots of kiwi fruit seedlings are significantly upregulated under waterlogging treatment [30]. This study revealed that the expression difference levels of six WRKY transcription factors gradually decreased in ZM22, BN206 and BN607 (Table 1), which indicated that the germination of ZM22, BN207 and BN607 seeds under the waterlogging treatment was negatively regulated by WRKY transcription factors. AP2/ERF plays an important role in the responses to external biotic and abiotic stresses [37-39]. SUB1A, an ERF transcription factor, can inhibit ethylene synthesis and the response of rice to gibberellin under waterlogging stress, reduce carbohydrate consumption and improve the waterlogging tolerance [40, 41]. Lee et al. [42] found that CIPK15 expression is induced by hypoxia and a lack of glucose during rice germination under waterlogging, which causes a series of potential downstream chain reactions by activating the energy and stress receptive factor SnRK1. This effect can enhance the expression of amylolytic enzyme genes and the synthesis of alcohol dehydrogenase, which would promote anaerobic respiration to ensure the provision of sufficient metabolic energy for cell growth. During this time, the coleoptile can also undergo rapid elongation. In this study, the expression difference levels of one AP2/ERF transcription factor (TraesCS1B02G392300) increased successively in ZM22, BN206 and BN607 (Table 1), which indicated that the expression level of the AP2/ ERF transcription factor could be used as an important reference index for evaluating the difference in waterlogging tolerance among the three wheat varieties. Further research on the regulatory mechanism of AP2/ERF transcription factors is needed in the future.

\section{Conclusions}

In this study, three wheat varieties with different waterlogging tolerances were subjected to waterlogging treatment. Analyses of the seed GRs and the changes in the embryo and endosperm structures revealed that BN607 exhibited the strongest waterlogging tolerance, followed by BN207 and ZM22. To further reveal the difference in waterlogging tolerance among the three wheat varieties during germination, a transcriptome analysis was performed, and the results identified a total of 2775 DEGs between the control and waterlogging treatments. Based on the correlations between the DEGs and the GR, 563 and 398 key genes that exhibited positive and negative correlations, respectively, were screened. GO and KEGG analyses showed that the waterlogging tolerance of the 
three wheat varieties was closely related to glycolysis, starch and sucrose metabolism and the lactose metabolism pathway and that $\mathrm{ADH}$ genes played an important role in the response to short-term waterlogging. These results will provide insights into the mechanistic regulation of some candidate genes and could thus reveal the molecular mechanisms underlying the waterlogging tolerance of wheat seeds during the germination stage.

\section{Methods}

\section{Experimental materials and treatment methods}

In this study, three wheat (Triticum aestivum L.) varieties, 'Zhoumai 22' (ZM22, waterlogging-intolerant), 'Bainong 207' (BN207, waterlogging-medium) and 'Bainong 607' (BN607, waterlogging-tolerant), were selected as the materials. ZM22 is a commercial winter wheat cultivar in Henan Province, China. The BN207 and BN607 seeds used in this study were cultivated by Prof. Xingqi Ou from the School of Life Science and Technology, Henan Institute of Science and Technology, Xinxiang, China. All the seeds were provided by the School of Life Science and Technology, Henan Institute of Science and Technology. Intact seeds with uniform sizes and no pests or diseases were selected for the experiment. The seeds were first disinfected with $5 \%$ hydrogen peroxide for $5 \mathrm{~min}$, washed three to five times with steam water, and submerged 12cm-diameter glass Petri dishes filled with $150 \mathrm{~mL}$ of sterilized deionized water, and the Petri dishes were covered with aluminium foil to minimize gas exchange.

Experimental treatment: For the waterlogging treatment, 50 plump seeds in glass Petri dishes were incubated at a constant temperature of $20^{\circ} \mathrm{C}$ in the dark for $72 \mathrm{~h}$. At the end of the waterlogging treatment, the seeds were transferred and germinated in a Petri dish containing one layer of filter paper and $10 \mathrm{~mL}$ of sterilized deionized water. Control treatment: Each variety was subjected to one control treatment (CK), which did not include any waterlogging. The seeds were germinated as in the waterlogging treatment. The experimental and control treatments included five replicates, and each replicate consisted of 50 seeds. All the seeds were grown in a growth chamber with $25^{\circ} \mathrm{C}, 75 \%$ relative humidity and a $16-\mathrm{h}$ light/8-h dark cycle. The GR of the seeds (the number of germinated seeds) after $72 \mathrm{~h}$ of germination was calculated. Seeds were considered germinated if the radicle and the hypocotyl had emerged at least $2 \mathrm{~mm}$ from the seed coat. After $72 \mathrm{~h}$ of germination, we removed the radicle and coleoptile of the seeds; part of the samples were used for analysis of the seed anatomy, and the other samples were used for transcriptomic sequencing.

\section{Measurement of the $\mathrm{GR}$ and $\mathrm{CH}$ of wheat seeds}

After $72 \mathrm{~h}$ of germination, the GR and the $\mathrm{CH}$ of the seeds in both the waterlogging and control treatments were measured.

\section{Structural anatomy of wheat seeds}

To assess the anatomy of wheat seeds, the seeds were fixed in FAA (38\% formaldehyde:glacial acetic acid:70\% alcohol = 1:1:18 volume ratio) solution, sliced and embedded in paraffin. The slices were then processed as follows: Fist, the sections were sequentially placed in xylene I for $20 \mathrm{~min}$, xylene II for $20 \mathrm{~min}$, absolute ethanol I for $5 \mathrm{~min}$, absolute ethanol II for $5 \mathrm{~min}$, 75\% alcohol for $5 \mathrm{~min}$, and tap water for $5 \mathrm{~min}$ to deparaffinize the paraffin sections until dehydration. Second, the slices obtained using an ultramicrotome (Ultracut R, Leica, Germany) were sequentially stained with periodic acid, Schiff's reagent, and naphthol yellow S (Wuhan Servicebio Technology Co., Ltd.). Third, the slices were dehydrated and mounted with anhydrous ethanol, xylene and neutral gum. Fourth, the slices were observed under a light microscope (DMLS, Leica, Solms, Germany) and photographed with a camera (TrueChrome II, Tucsen, China). Photoshop and Image-Pro Plus 6.0 (Media Cybernetics, Inc., Rockville, MD, USA) were used to count the numbers of amyloplasts based on the micrographs (three replicate seeds and 10 micrographs of each seed).

\section{Total RNA isolation, RNA library construction and transcriptome sequencing}

To explore the response of wheat seeds to waterlogging stress, a transcriptome analysis of the seeds of three wheat varieties, 'ZM22', 'BN207' and 'BN607', under the waterlogging and control treatments was performed. A total of 18 samples (C72_ZM22, C72_BN207, C72_ BN607, W72_ZM22, W72_BN207, and W72_BN607; each treatment was performed in triplicate) were first obtained by extracting total RNA from the samples using the TRIzol kit (Invitrogen, CA, USA). Total RNA was removed with RNase-free DNase I (Takara, Tokyo, Japan) to avoid genomic DNA contamination. To ensure that transcriptome sequencing was performed using high-quality samples, the purity, concentration, and integrity of RNA samples were determined using a NanoDrop, a Qubit 2.0, and an Agilent 2100, respectively. First, the mRNA was enriched with oligo (dT) magnetic beads, and fragmentation buffer was added to randomly disrupt the mRNA. The first cDNA strand was synthesized using mRNA as a template and a six-base random primer (random hexamers). To synthesize the second cDNA strand, the first cDNA strand was combined with buffer, dNTPs, RNase $\mathrm{H}$, and DNA polymerase I. The purified double-stranded cDNA was subjected to end repair, A tailed and ligated to the sequencing adapter. Finally, fragment size selection was performed using AMPure XP beads, and a cDNA library was enriched by PCR. After the library was constructed, the concentration of the library and the insert size were detected using a Qubit 2.0 and an Agilent 2100, respectively. The 
average insert size of the paired-end libraries was $150 \mathrm{bp}$ $( \pm 50 \mathrm{bp})$. After inspection of the library quality, transcriptome sequencing was performed using an Illumina NovaSeq $^{\text {tw }} 6000$ (LC Sciences, San Diego, CA, USA) according to the standard protocols of the LC-Bio Technology Co., Ltd. (Hang Zhou, Zhejiang Province, China).

\section{Normalization and annotation of sequencing data}

To ensure accurate and credible results, we preprocessed the raw data, which included the removal of sequencing adapters and low-quality sequencing data. The valid data were aligned to the reference genome of wheat (https:// urgi.versailles.inra.fr/download/iwgsc/IWGSC_RefSeq_ Assemblies/v1.0/), and statistical information was obtained based on gene location information specified by genome annotation files (GTF and GFF).

\section{Sequencing sequence statistics and quality control}

The raw data generated by sequencing needs to be preprocessed. We used cutadapt to filter out unqualified sequences and obtain clean data [43]. The specific processing steps were as follows: (1) removing adaptercontaining reads; (2) removing reads with a content ratio of $\mathrm{N}$ ( $\mathrm{N}$ represents a base that cannot be determined) that is greater than $5 \%$ of the reads; (3) removing lowmass reads (mass value of $\mathrm{Q} \leq 10$ bases accounting for more than $20 \%$ of the entire reads); and (4) analysing the amount of raw sequencing data, an effective amount of sequencing data, and the Q20, Q30, and GC contents.

\section{Analysis of DEGs and gene function annotation}

The aligned reads from each sample were assembled using StringTie. The transcriptomes of all the samples were then reused to construct a comprehensive transcript utilising a Perl script. After the final generation of transcripts, StringTie and EdgeR were used to estimate the expression levels of all the transcripts. The expression levels of the genes were characterized by calculating FPKM values (fragments per kilobase of exon model per million mapped reads) [44], which eliminated the influences of the gene length and sequencing level on the calculation of gene expression. The DEGs in 'ZM22', 'BN207' and 'BN607' were identified by comparing the waterlogging and control treatments. The differentially expressed mRNAs with $\log 2$ (fold change) $>1$ or $\log 2$ (fold change) $<-1$ and with statistical significance $(p$ value $<0.05)$ were identified using the $R$ package Ballgown [45]. The fold change represents the ratio of expression under the waterlogging and to that under the control treatment. An expression level dominance analysis was performed using EdgeR software [46], and a cluster analysis of the DEGs was performed using clustering software and Java Treeview. Gene functional annotations were based on the pear genome database and mapped to GO terms. GO enrichment analysis was performed using WEGO, and KEGG pathways were identified according to $p$-values and adjusted $\mathrm{q}$ values through a BLAST search against the KEGG database and subsequent mapping to KEGG pathways [47-52].

\section{Total RNA extraction, reverse transcription and qRT-PCR assays}

Based on the target gene sequences, 10 gene-specific primer pairs were designed (Table S6). The RNA samples used for qRT-PCR analysis were aliquots of the samples used in the RNA-Seq experiments. The qRT-PCR assays were performed with the Primer Script RT Reagent Kit (Takara, Dalian, China) and the reference gene $18 \mathrm{~S}$ (Gene ID: AJ272181.1) using cDNA from the transcriptome samples as the template. The total reaction volume for each qRT-PCR was $20 \mu \mathrm{L}$, which comprised $10 \mu \mathrm{L}$ of SYBR Green PCR SuperMix (Vazyme Biotech Co., Ltd., Nanjing, China), $0.4 \mu \mathrm{L}$ of each primer, $2 \mu \mathrm{L}$ of cDNA, $0.4 \mu \mathrm{L}$ of passive reference dye and $6.8 \mu \mathrm{L}$ of doubledistilled water. The PCR conditions were as follows: $95^{\circ} \mathrm{C}$ for $10 \mathrm{~s}$ and 40 cycles of $95^{\circ} \mathrm{C}$ for $5 \mathrm{~s}$ and $60^{\circ} \mathrm{C}$ for $30 \mathrm{~s}$. the qRT-PCRs were performed using an ABI Step One Plus. The qRT-PCR data were obtained from technical replicates with error bars and are presented as the means \pm SEs $(n=3)$. One sample constitutes a mixture of three seeds. The relative expression was calculated using the $2^{-\Delta \Delta C t}$ method [53].

\section{Statistical analysis}

All statistical analyses were performed with SPSS 18.0 (SPSS Inc., Chicago, IL, USA) and Microsoft Excel (Microsoft Corporation, Redmond, WA, USA). The data were analysed by one-way analysis of variance (ANOVA). Mean separations were performed by Duncan's multiple range tests. OriginPro 8.1 (Origin Inc., Chicago, IL, USA) was used to draw the figures. Differences with $p<0.05$ were considered significant.

\section{Supplementary information}

Supplementary information accompanies this paper at https://doi.org/10. 1186/s12863-020-00901-y.

Additional file 1: Table S1. Summary of the RNA-Seq data collected from the seeds of three wheat varieties (ZM22, BN207 and BN607) under the waterlogging and control treatments.

Additional file 2: Table S2. Summary of the genome alignment distribution of the RNA-Seq data.

Additional file 3: Table S3. Summary of the GO results of the DEGs. Additional file 4: Table S4. KEGG enrichment analysis of the DEGs. Additional file 5: Table S5. Statistics of DEGs that exhibit different expression patterns.

Additional file 6: Table S6. List of primer sequences used for qPCR analysis.

Additional file 7: Table S7. Cluster analysis of the DEGs. 
Additional file 8: Figure S1. Principal component analysis (PCA) $f$ the transcription levels in the seeds of three wheat varieties (ZM22, BN207 and BN607) under the waterlogging and control treatments. ZM22: Zhoumai 22, BN207: Bainong 207, BN607: Bainong 607. W72_ZM22, W72 BN207 and W72 BN607 refer to ZM22, BN207 and BN607 under the waterlogging treatment, respectively. C72_ZM22, C72_BN207 and C72_BN607 refer to ZM22, BN207 and BN607 under the control treatment, respectively.

Additional file 9: Figure S2. GO enrichment of DEGs in the seeds of three wheat varieties (ZM22, BN207 and BN607) under the waterlogging and control treatments. W72_ZM22, W72_BN207 and W72_BN607 refer to ZM22, BN207 and BN607 under the waterlogging treatment, respectively. C72_ZM22, C72_BN207 and C72_BN607 refer to ZM22, BN207 and BN607 under the control treatment, respectively.

Additional file 10: Figure S3. KEGG enrichment factor analysis of DEGs in the seeds of three wheat varieties (ZM22, BN207 and BN607) under the waterlogging and control treatments. W72_ZM22, W72_BN207 and W72_BN607 refer to ZM22, BN207 and BN607 under the waterlogging treatment, respectively. C72_ZM22, C72_BN207 and C72_BN607 refer to ZM22, BN207 and BN607 under the control treatment, respectively.

Additional file 11: Figure S4. RT-qPCR validations of the RNA-seq data. Expression profiling of 10 candidate genes of the seeds of three wheat varieties (ZM22, BN207 and BN607) under the waterlogging and control treatments. W72 ZM22, W72 BN207 and W72_BN607 refer to ZM22, BN207 and BN607 under the waterlogging treatment, respectively. C72_ZM22, C72_BN207 and C72_BN607 refer to ZM22, BN207 and BN607 under the control treatment, respectively.

\section{Abbreviations}

ZM22: Zhoumai 22; BN207: Bainong 207; BN607: Bainong 607;

DEGs: Differentially Expressed Genes; ADH: Alcohol Dehydrogenase; GO: Gene Ontology; mRNA: Messenger RNA; KEGG: Kyoto Encyclopedia of Genes and Genomes; PCA: Principal Component Analysis; qRT-

PCR: Quantitative Real-time PCR; FPKM: Fragments Per Kilobase Per Million Mapped Reads; NCBI: National Center for Biotechnology Information; PDC: Pyruvate Decarboxylase; LDH: Lactate Dehydrogenase; GR: Germination Rate; CH: Coleoptile Height; FAA: 38\% Formaldehyde-Acetic acid-70\% Alcohol

\section{Acknowledgements}

The authors thank the LC-Bio Technology Co., Ltd. (Hang Zhou, Zhejiang Province, China), for the assistance provided with the data processing and bioinformatic analysis. We are indebted to Prof. Xingqi Ou for the support provided with the experimental seed materials.

\section{Authors' contributions}

CWS, JPY, XJR, XLC and XOO conceived, designed and supervised the experiments; CWS and JPY wrote the manuscript; CWS, HQ, ZJW, and YHL performed the experiments; CWS, JPY, and XL provided support to the lab experiment and data analyses; and CWS, YZ, FW, and JPY analysed the data. All the authors read and approved the manuscript.

\section{Funding}

This work was funded by the Major Science and Technology Project in Xinxiang (ZD18007), the fund of the National Key R\&D Program of China (2017YFD0201700), and the Scientific Research Foundation for High-level Talent (2017034). These funding sources provided support for the data collection and manuscript writing.

\section{Availability of data and materials}

The datasets supporting the results described in this article are included within the article and its additional file. All raw sequence reads have been deposited in NCBI's Gene Expression Omnibus and are accessible under the GEO Series accession number GSE144554 (https://www.ncbi.nlm.nih.gov/ geo/query/acc.cgi?acc=GSE144554).

\section{Ethics approval and consent to participate}

Three wheat cultivars, 'Zhoumai 22' (ZM22), 'Bainong 207' (BN207) and 'Bainong 607' (BN607), were used in the present study. ZM22 originated from a cross of three breeding lines, namely Zhoumai 12, Wenmai 6 and Zhoumai 13. The BN207 and BN607 seeds used in this study were cultivated by Prof. Xingqi Ou from the School of Life Science and Technology, Henan Institute of Science and Technology, Xinxiang, China.

\section{Consent for publication}

Not applicable.

\section{Competing interests}

The authors declare that they have no competing interests.

\section{Author details}

${ }^{1}$ School of Resources and Environmental Sciences, Henan Institute of Science and Technology, Xinxiang 453003, China. ${ }^{2}$ School of Horticulture and Landscape Architecture, Henan Institute of Science and Technology, Xinxiang 453003, China. ${ }^{3}$ Xinxiang Nongle Seed Industry Co. Ltd, Xinxiang 453003, China. ${ }^{4}$ School of Life Science and Technology, Henan Institute of Science and Technology, Xinxiang 453003, China.

\section{Received: 22 April 2020 Accepted: 16 August 2020}

Published online: 28 August 2020

\section{References}

1. Huang B, Johnson JW, Box JE, NeSmith DS. Root characteristics and hormone activity of wheat in response to hypoxia and ethylene. Crop Sci. 1997;37:812-8

2. Herzog M, Striker GG, Colmer TD, Pedersen O. Mechanisms of waterlogging tolerance in wheat-a review of root and shoot physiology. Plant Cell Environ. 2016;39:1068-86.

3. Huang B, Johnson JW, Nesmith S, Bridges DC. Growth, physiological and anatomical responses of two wheat genotypes to waterlogging and nutrient supply. J Exp Bot. 1994;45:193-202.

4. Huang B, Johnson JW, NeSmith DS, Bridges DC. Nutrient accumulation and distribution of wheat genotypes in response to waterlogging and nutrient supply. Plant Soil. 1995;173:47-54.

5. Li C, Jiang D, Wollenweber B, Li Y, Dai T, Cao W. Waterlogging pretreatment during vegetative growth improves tolerance to waterlogging after anthesis in wheat. Plant Sci. 2011;180:672-8.

6. Bassu S, Asseng S, Motzo R, Giunta F. Optimising sowing date of durum wheat in a variable Mediterranean environment. Field Crop Res. 2009;111: 109-18.

7. Arduini I, Orlandi C, Ercoli L, Masoni A. Submergence sensitivity of durum wheat, bread wheat and barley at the germination stage. Ital J Agron. 2016; 11:100.

8. Setter TL, Waters I. Review of prospects for germplasm improvement for waterlogging tolerance in wheat, barley and oats. Plant Soil. 2003;253:1-34.

9. Bailey-Serres J, Voesenek LACJ. Flooding stress: acclimations and genetic diversity. Annu Rev Plant Biol. 2008;59:313-39.

10. Colmer TD, Voesenek LACJ. Flooding tolerance: suites of plant traits in variable environments. Funct Plant Biol. 2009;36:665-81.

11. Perata P, Pozueta-Romero J, Akazawa T, Yamaguchi J. Effect of anoxia on starch breakdown in rice and wheat seeds. Planta. 1992;188:611-8.

12. Malik Al, Colmer TD, Lambers H, Setter TL, Schortemeyer M. Short-term waterlogging has long-term effects on the growth and physiology of wheat. New Phytol. 2002;153:225-36.

13. Herzog M, Fukao T, Winkel A, Konnerup D, Lamichhane S, Alpuerto JB, et al. Physiology, gene expression, and metabolome of two wheat cultivars with contrasting submergence tolerance. Plant Cell Environ. 2018:41:1632-44.

14. Winkel A, Herzog M, Konnerup D, Floytrup AH, Pedersen O. Flood tolerance of wheat-the importance of leaf gas films during complete submergence. Funct Plant Biol. 2017:44:888-98.

15. Hsu SK, Tung CW. RNA-Seq analysis of diverse rice genotypes to identify the genes controlling coleoptile growth during submerged germination. Front Plant Sci. 2017:8:762.

16. Yang $Y$, Chen $X Y$, Ran $L P, W u$ Y F, Yu XR, Dong ZD, et al. Formation of protein bodies and the response to nitrogen in different positions during wheat endosperm development. J Plant Biol. 2019:62:274-85.

17. Zhou Q, Huang M, Huang X, Liu J, Wang X, Cai J, et al. Effect of postanthesis waterlogging on biosynthesis and granule size distribution of starch in wheat grains. Plant Physiol Biochem. 2018;132:222-8. 
18. Lin Z, Qi Y, Liu D, Mao FF, Deng XY, Li JW, et al. The effects of different pharmacological agent treatments on ROS accumulation and functional protein activities in wheat endosperm PCD. Acta Physiol Plant. 2019;41(5):62.

19. Cannell RQ, Belford RK, Gales K, Dennis CW, Prew RD. Effects of waterlogging at different stages of development on the growth and yield of winter wheat. J Sci Food Agric. 1980;31:117-32.

20. Takeda K. Tolerance to pre-germination flooding in the world collection of barley varieties. Barley Genet. 1987;1:735-40.

21. Luan H, Guo B, Pan Y, Lv C, Shen H, Xu R. Morpho-anatomical and physiological responses to waterlogging stress in different barley ( Hordeum vulgare L.) genotypes. Plant Growth Regul. 2018;85:399-409.

22. Liu Y, Han C, Deng X, Liu D, Liu N, Yan Y. Integrated physiology and proteome analysis of embryo and endosperm highlights complex metabolic networks involved in seed germination in wheat (Triticum aestivum L.). J Plant Physiol. 2018;229:63-76.

23. Tosi P, Parker M, Gritsch CS, Carzaniga R, Martin B, Shewry PR. Trafficking of storage proteins in developing grain of wheat. J Exp Bot. 2009;60:979-91.

24. Komatsu S, Sugimoto T, Hoshino T, Nanjo Y, Furukawa K. Identification of flooding stress responsible cascades in root and hypocotyl of soybean using proteome analysis. Amino Acids. 2010;38:729-38.

25. Nanjo Y, Maruyama K, Yasue H, Yamaguchi-Shinozaki K, Shinozaki K, Komatsu S. Transcriptional responses to flooding stress in roots including hypocotyl of soybean seedlings. Plant Mol Biol. 2011;77:129-44.

26. Tougou M, Hashiguchi A, Yukawa K, Nanjo Y, Hiraga S, Nakamura T, et al. Responses to flooding stress in soybean seedlings with the alcohol dehydrogenase transgene. Plant Biotechnol. 2012;29:301-5.

27. Branco-Price C, Kaiser KA, Jang CJ, Larive CK, Bailey-Serres J. Selective mRNA translation coordinates energetic and metabolic adjustments to cellular oxygen deprivation and reoxygenation in Arabidopsis thaliana. Plant J. 2008; 56:743-55

28. Komatsu S, Thibaut D, Hiraga S, Kato M, Chiba M, Hashiguchi A, et al. Characterization of a novel flooding stress-responsive alcohol dehydrogenase expressed in soybean roots. Plant Mol Biol. 2011;77:309-22.

29. Liu Z. Adams KL expression partitioning between genes duplicated by polyploidy under abiotic stress and during organ development. Curr Biol. 2007:17:1669-74.

30. Zhang J, Huang S, Mo Z, Xuan JP, Jia XD, Wang G, et al. De novo transcriptome sequencing and comparative analysis of differentially expressed genes in kiwifruit under waterlogging stress. Mol Breed. 2015;35: 208.

31. Howell KA, Narsai R, Carroll A, Ivanova A, Lohse M, Usadel B, et al. Mapping metabolic and transcript temporal switches during germination in Rice highlights specific transcription factors and the role of RNA instability in the germination process. Plant Physiol. 2008;149:961-80.

32. Katonoguchi $\mathrm{H}$, Morokuma M. Ethanolic fermentation and anoxia tolerance in four rice cultivars. J Plant Physiol. 2007;164:168-73.

33. Vodnik D, Strajnar P, Jemc S, Macek I. Respiratory potential of maize (Zeo mays L.) roots exposed to hypoxia. Environ Exp Bot. 2009;65:107-10.

34. Trethowan R, Singh RP, Huertaespino J, Crossa J, Van Ginkel M. Coleoptile length variation of near-isogenic Rht lines of modern CIMMYT bread and durum wheats. Field Crop Res. 2001;70:167-76.

35. Huang J, Toyofuku K, Yamaguchi J, Akita S. Expression of a-amylase and the RAmy1A gene in Rice ( L.) during seed germination, and its relationship with coleoptile length in submerged soil. Plant Prod Sci. 2000;3:32-7.

36. Rushton DL, Tripathi P, Rabara RC, Lin J, Ringler P, Boken AK, et al. WRKY transcription factors: key components in abscisic acid signalling. Plant Biotechnol J. 2012;10:2-11.

37. Zhuang J, Peng RH, Cheng ZMM, Zhang J, Cai B, Zhang Z, et al. Genomewide analysis of the putative AP2/ERF family genes in Vitis vinifera. Sci Hortic. 2009;123:73-81.

38. Li MY, Wang F, Jiang Q, Li R, Ma J, Xiong AS. Genome-wide analysis of the distribution of AP2/ERF transcription factors reveals duplication and elucidates their potential function in Chinese cabbage (Brassica rapa ssp. pekinensis). Plant Mol Biol Report. 2013;31:1002-11.

39. Yu Y, Duan XB, Ding XD, Chen C, Zhu D, Yin K, et al. L, HQ, Liu BD, Zhu YM. A novel AP2/ERF family transcription factor from Glycine soja, GsERF71, is a DNA binding protein that positively regulates alkaline stress tolerance in Arabidopsis. Plant Mol Biol. 2017;94:509-30.

40. Fukao T, Xu K, Ronald PC, Bailey-Serres J. A variable cluster of ethylene response factor-like genes regulates metabolic and developmental acclimation responses to submergence in rice. Plant Cell. 2006;18:2021-34.
41. Fukao T, Yeung E, Bailey-Serres J. The submergence tolerance regulator SUB1A mediates crosstalk between submergence and drought tolerance in rice. Plant Cell. 2011;23:412-27.

42. LEE KW, Chen PW, YU SM. Metabolic adaptation to sugar $/ \mathrm{O}_{2}$ deficiency for anaerobic germination and seedling growth in rice. Plant Cell Environ. 2014; 37:2234-44

43. Andrews S. FastQC: a quality control tool for high throughput sequence data; 2010. http://www.bioinformatics.babraham.ac.uk/projects/fastqc. Accessed 6 Oct 2011.

44. Pertea M, Pertea GM, Antonescu CM, Chang T-C, Mendell JT, Salzberg SL. StringTie enables improved reconstruction of a transcriptome from RNA-seq reads. Nat Biotechnol. 2015;33(3):290-5.

45. Frazee AC, Pertea G, Jaffe AE, Langmead B, Salzberg SL, Leek JT. Ballgown bridges the gap between transcriptome assembly and expression analysis. Nat Biotechnol. 2015:33(3):243.

46. Rapp RA, Udall JA, Wendel JF. Genomic expression dominance in allopolyploids. BMC Biol. 2009;7(1):18

47. Ashburner M, Ball CA, Blake JA, Botstein D, Butler H, Cherry JM, et al. Gene ontology: tool for the unification of biology. Nat Genet. 2000;1:9-25.

48. Tatusov RL. The COG database: a tool for genome-scale analysis of protein functions and evolution. Nucleic Acids Res. 2000;1(28):33-6.

49. Apweiler R, Bairoch A, Wu CH, Barker WC, Boeckmann B, Ferro S, et al. UniProt: the universal protein knowledgebase. Nucleic Acids Res. 2004;32: D115-9.

50. Kanehisa M, Goto S, Kawashima S, Okuno Y, Hattori M. The KEGG resource for deciphering the genome. Nucleic Acids Res. 2004;32:D277-80.

51. Young MD, Wakefield MJ, Smyth GK, Oshlack A. Gene ontology analysis for RNA-seq: accounting for selection bias. Genome Biol. 2010;11:R14.

52. Finn RD. Pfam: the protein families database. Nucleic Acids Res. 2014;42: D30-D222.

53. Livak KJ, Schmittgen TD. Analysis of relative gene expression data using real-time quantitative PCR and the 2(-Delta Delta $C(T))$ method. Methods. 2001;25:402-8.

\section{Publisher's Note}

Springer Nature remains neutral with regard to jurisdictional claims in published maps and institutional affiliations.

\section{Ready to submit your research? Choose BMC and benefit from:}

- fast, convenient online submission

- thorough peer review by experienced researchers in your field

- rapid publication on acceptance

- support for research data, including large and complex data types

- gold Open Access which fosters wider collaboration and increased citations

- maximum visibility for your research: over $100 \mathrm{M}$ website views per year

At $\mathrm{BMC}$, research is always in progress.

Learn more biomedcentral.com/submissions 